\title{
A Microwave Fourier Transform Technique using Double Resonance Modulation
}

\author{
W. Stahl, J. Gripp, N. Heineking, and H. Dreizler \\ Abteilung Chemische Physik im Institut für Physikalische Chemie der Universität Kiel \\ Z. Naturforsch. 42 a, 392-394 (1987); received December 31, 1986 \\ We present a further modification of the double resonance technique in microwave FOURIER \\ transform spectroscopy. The method is promising for the search of double resonances. It is \\ demonstrated by three examples.
}

In frequency domain microwave (MW) spectroscopy double resonance (DR) methods have proved to be very valuable $[1-3]$. A survey on the use of DR in the frequency domain is given in [3].

Some years ago we observed that DR effects can also be produced in the time domain by microwave FOURIER transform (MWFT) spectroscopy [4]. In this first experiment a continuous pump radiation was used. We described the experiment in terms of the density matrix formalism for a three-level system. Under the influence of pump radiation spectral lines split into doublets, as has also been observed in the frequency domain.

In a following paper [5] a pulsed pump radiation was applied prior to the polarization of the molecular ensemble. In this case the occupation number difference of the signal transition is modified. The change in intensity of the molecular signal was observed.

Later we succeeded in showing [6] that a two dimensional DR spectroscopy is also possible, in which one signal pulse and two pump pulses are used. This method is promising but time consuming because each experiment consists of a series of some hundred individual recordings, in which the delay between the two pump pulses is varied.

In this paper we report a new type of experiment carried out according to the schedule shown in Figure 1. Due to the alternating addition and subtraction the transient emission signals of transitions not influenced by the DR effect compensate. An example of the transient emission without and

Reprint request to Prof. Dr. Helmut Dreizler, Institut für Physikalische Chemie, Universität Kiel, Ludewig-MeynStr. 8, D-2300 Kiel. under influence of the pump is given in Fig. 1 of [7]. Only those transitions effected by DR show up. In this way a complex spectrum with many transitions is simplified, as is shown in Figs. 2 to 4 . Further the coherent perturbations also cancel. The theory given in the preceeding papers is sufficient also for this type of DR experiment. Therefore it is not repeated here.

Figure 2 a shows a $10 \mathrm{MHz}$ section of the rotational spectrum of isopropyl iodide, $\mathrm{CH}_{3} \mathrm{CHICH}_{3}$, near $4950 \mathrm{MHz}$ recorded with the usual MWFTtechnique. The weak c-type transition $J K_{-} K_{+}=$ $313-303, F=11 / 2-11 / 2$ at $4945.84 \mathrm{MHz}$ is indicated.

Figure $2 \mathrm{~b}$ shows the same spectral region under almost resonant pump modulation. The pump transition is $413-313,13 / 2-11 / 2$, a strong a-type transition at $15271.84 \mathrm{MHz}$.

Figure $3 \mathrm{~b}$ demonstrates that in favourable cases the complex line shape of the pump modulation spectra can be seen. The line $J K_{-} K_{+}=514-515$, $F=13 / 2-13 / 2$, at $5521.14 \mathrm{MHz}$ consists of the original transition uneffected by the pump and the transition split by influence of the pump.

Figure $3 \mathrm{a}$ shows the normal spectrum for comparison. The pump transition is a very weak c-type transition $J K_{-} K_{+}=524-514 F=13 / 2-13 / 2$. By this measurement the pump transition could be located.

The possibility of off-resonant pumping is illustrated in Figure 4. A section of $50 \mathrm{MHz}$ of the rotational spectrum of perfluoro pyridine, $\mathrm{C}_{5} \mathrm{~F}_{5} \mathrm{~N}$, near $5915 \mathrm{MHz}$ is displayed. The transition $J K_{-} K_{+}$ $=854-845$ at $5916.0 \mathrm{~Hz}$ is marked.

The nuclear hyperfine structure is unresolved. Figure $4 \mathrm{a}$ is taken without pump radiation.

0340-4811/87 / 0400-0392 \$01.30/0. - Please order a reprint rather than making your own copy. 


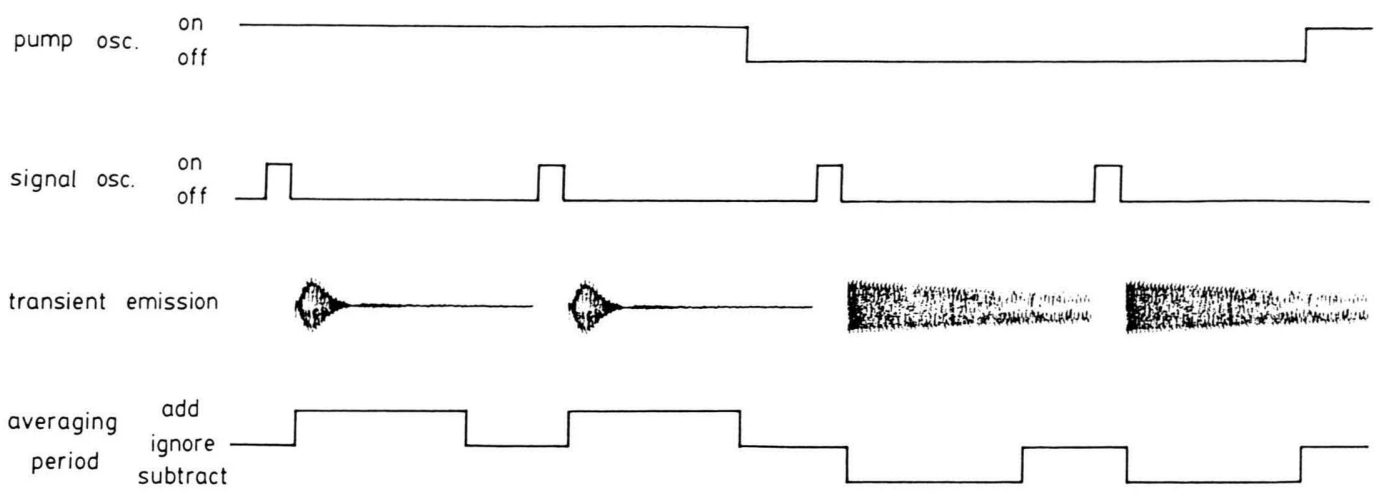

Fig. 1. Time schedule for MWFT double resonance modulation spectroscopy.

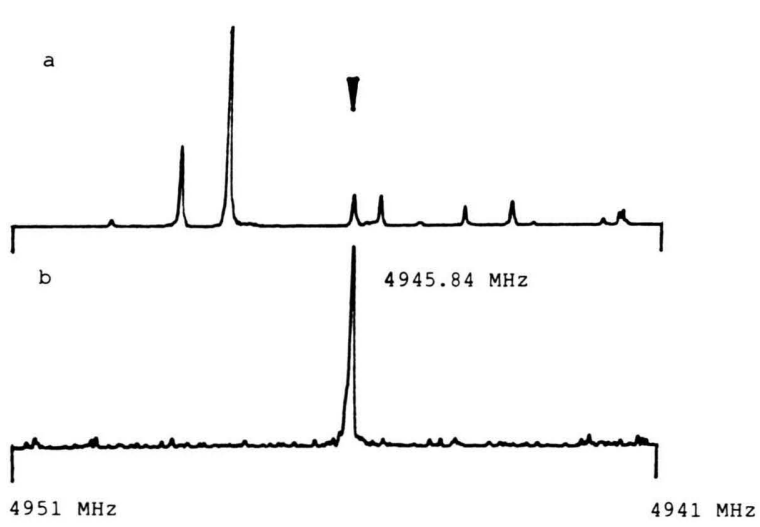

Fig. 2. A section from $4951-4941 \mathrm{MHz}$ of the rotational power spectrum of $\mathrm{CH}_{3} \mathrm{CHICH}_{3} . T=-40^{\circ} \mathrm{C}, p=0.1 \mathrm{~Pa}$. Sample intervall $20 \mathrm{~ns}, 1.6 \times 10^{6}$ averaging cycles, 3072 zeros supplemented prior to FOURIER transformation. a) Recorded without pump radiation. b) Recorded with pump radiation modulation at $15271.8 \mathrm{MHz}, 0.5 \mathrm{~mW}$ mean pump power at the sample cell entrance.

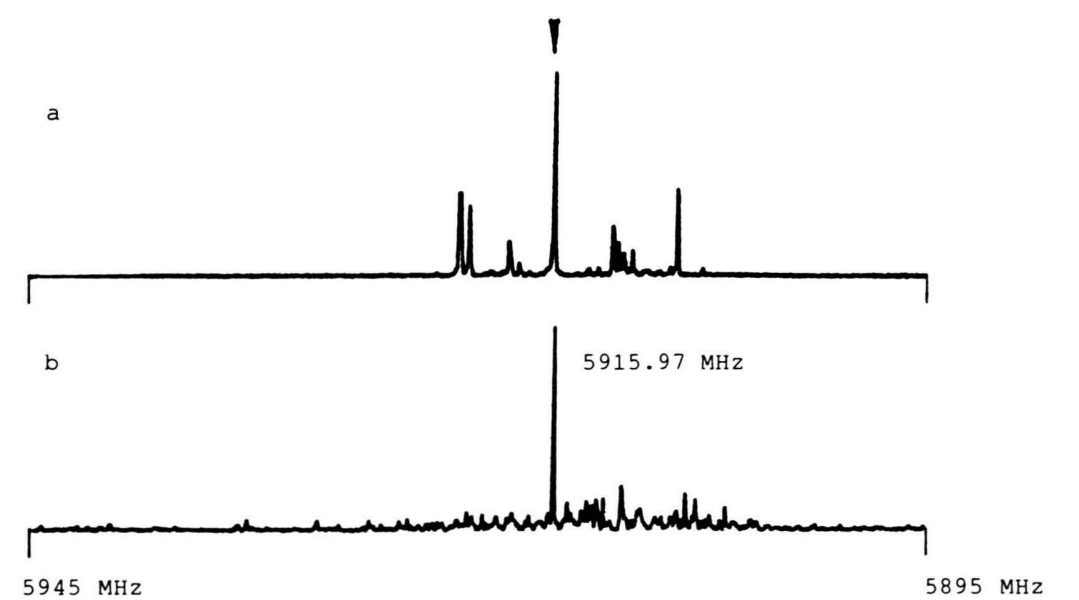

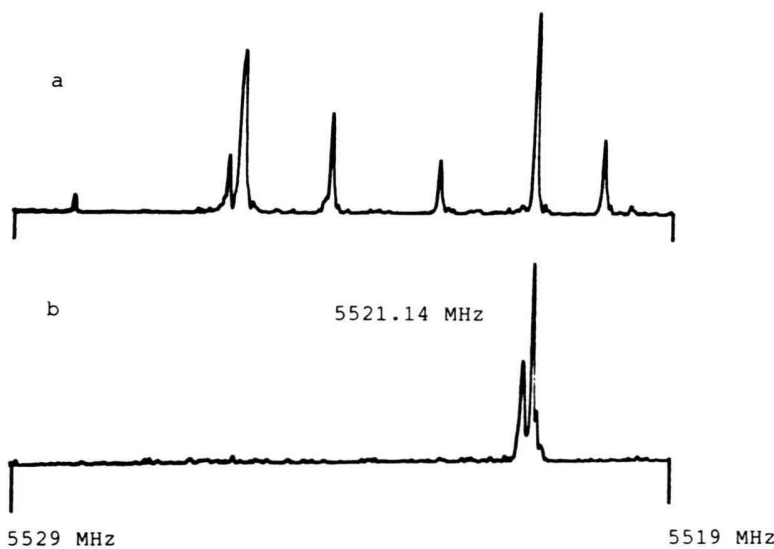

Fig. 3. A section from $5529-5519 \mathrm{MHz}$ of the rotational power spectrum of $\mathrm{CH}_{3} \mathrm{CHICH}_{3}$, experimental conditions see Figure 2. a) Recorded without pump radiation. b) Recorded with pump radiation at $15405.7 \mathrm{MHz}, 25 \mathrm{~mW}$ mean pump power at the sample cell entrance and with reduced pressure of $0.05 \mathrm{~Pa}$.
Fig. 4. A section from $5945-5895 \mathrm{MHz}$ of the rotational power spectrum of $\mathrm{C}_{5} \mathrm{~F}_{5} \mathrm{~N}$. $T=-30^{\circ} \mathrm{C}, p=0.4 \mathrm{~Pa}$, sample intervall $10 \mathrm{~ns}, 10^{6}$ averaging cycles. A) Recorded without pump radiation. b) Recorded with pump radiation at $15090.0 \mathrm{MHz}$, $3 \mathrm{~W}$ mean pump power. The pump transition $J K_{-} K_{+}=$ $936-845$ is at $15272.30 \mathrm{MHz}$. 


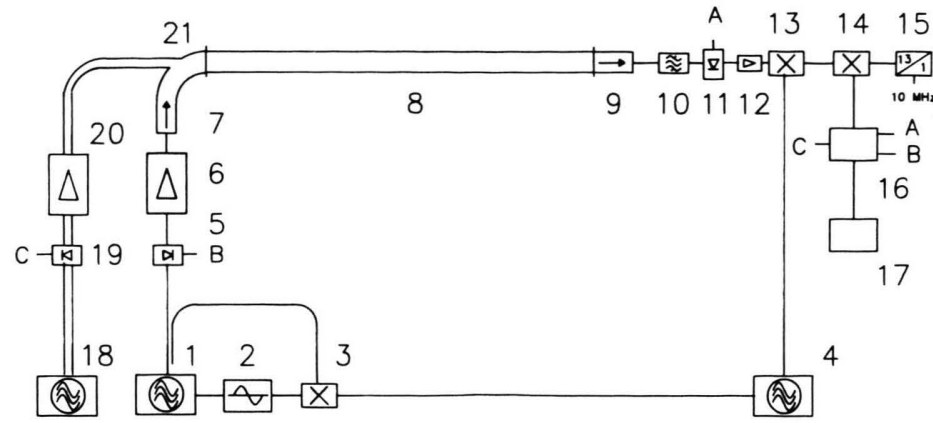

Fig. 5. General set up of a MW spectrometer with FOURIER transform technique for double resonance modulation. See text.
Figure $4 \mathrm{~b}$ shows the same spectral region with pump modulation and $182 \mathrm{MHz}$ pump off-resonance. The magnitude of off-resonance compares to that experienced in cw MW-spectroscopy.

We used a spectrometer which follows the general set up given in Figure 5.

A signal oscillator 1 is phase stabilized to a phase stabilized local oscillator 4 by a mixer 3 and synchronizer 2 . The continuous (cw) microwave of 1 is pulse modulated by a pair of PIN switches 5 and amplified by a travelling wave tube amplifier (TWTA) 6 followed by a PIN switch. Via an isolator 7 the polarizing pulses are fed into the sample cell 8 . The isolators 7 and 9 reduce pulse reflections. The PIN switch 11 shields the detection system consisting of a MW amplifier 12 and MW mixer with IF preamplifier 13. By the IF mixer 14 and the IF local oscillator 15 the signal is transferred to a band around $30 \mathrm{MHz}$, which is processed by the averager 16 [8] and computer 17. This usual set up of a MWFT-spectrometer [9] is supplemented by a phase stabilized pump oscillator 18. The switch 19 produces the modulation of the pump microwave. The pump microwave is amplified by a TWTA 20 and fed into the sample cell 8 by a straight waveguide connected to an E-bend 21. A low pass or band pass filter 10 shields the detection system from the pump MW radiation. All frequencies of the

[1] J. G. Baker, Microwave-Microwave Double Resonance, G. W. Chantry (Ed.), Academic Press, New York 1979, pp. $65-121$.

[2] O. L. Stiefvater, Z. Naturforsch. 30 a, 1742 (1975).

[3] W. Gordy and R. L. Cook, Microwave Molecular Spectra, J. Wiley, New York 1984, 273-281 and citations herein.

[4] H. Dreizler, E. Fliege, H. Mäder, and W. Stahl, Z. Naturforsch. 37 a, 1266 (1982). system are derived from a $10 \mathrm{MHz}$ standard. The experiment control is incorporated in 16 .

This type of DR was performed with the signal radiation from $5-8 \mathrm{GHz}(\mathrm{J}$-band) and $8-12.4 \mathrm{GHz}$ (X-band) and with the pump radiation from $12.4-18 \mathrm{GHz}$ (Ku-band) and $18-26 \mathrm{GHz}$ (K-band) respectively. This selection of frequency ranges is imposed by the problem of feeding the pump radiation into the sample cell without interference with the performance of the MWFT spectrometer. The different frequency ranges of pump and signal allow easier separation of the pump modulation in front of the detection system. The advantage of this method is that it works also with a widely offresonant pump frequency (dependent on pump radiation power up to about hundred $\mathrm{MHz}$ ). This is important for searching double resonances in an unassigned spectrum. Presently we use the technique to confirm the tentative assignment of spectral lines. We are going to extend it to other frequency ranges and to use it for the assignment of spectra.

We thank the members of our group for help and discussions and the members of our mechanics workshop for the construction of special MW parts. The funds were provided by the Deutsche Forschungsgemeinschaft, the Fond der Chemie, and the Land Schleswig-Holstein.

[5] G. Bestmann and H. Dreizler, Z. Naturforsch. 38 a, 452 (1983).

[6] W. Stahl, E. Fliege, and H. Dreizler, Z. Naturforsch. 39 a, 858 (1984).

[7] W. Stahl and H. Dreizler, Z. Naturforsch. 40 a, 1096 (1985).

[8] G. Bestmann, Thesis Kiel 1984.

[9] E. Fliege and H. Dreizler, Z. Naturforsch. 42 a, 72 (1987). 